\title{
Practical Analysis of the Properties of Nanoscale Electronic Elements Aimed at their Application when Designing Parallel Architecture Computing Systems
}

\author{
M.V. Makarov* \\ Murom Institute of Vladimir State University, 23, Orlovskaya st., 602264 Murom, Russia
}

(Received 22 April 2016; published online 03 October 2016)

\begin{abstract}
This article presents an approach to the practical analysis of nanomaterials which determine the reliability parameters of nanoscale electronic hardware components when they are used in developing faulttolerant high-performance computing systems. We propose a methodology of theoretical and experimental study of the reliability values of the memristor models used as the synaptic connections of an artificial neural network that approximate a differential equation.
\end{abstract}

Keywords: Nanoscale electronics, Properties of nanomaterials, Memristors, Parallel computing, Fault tolerance.

\section{INTRODUCTION}

A need in the further efficient development of nanotechnologies and, in particular, nanoscale engineering has resulted in a new direction forming in the field of information technologies - nanoscale information technologies [1].

Some recent research shows that nanoscale information technologies can be used as an indispensable tool during one of the most important stages in the practical application of nanoparticles, nanoscale materials and nanoscale systems. This stage is considered as the study of their properties without separation from the future functional ownership. In other words, this stage enables us to answer the following questions: which properties of the system elements are formed by the system's performance characteristics and how does it happen when this system runs on a real time basis?

The analysis of the properties of nanoscale objects with the use of information technologies includes at least three main phases: object modeling, object display and experimental data analysis. Each phase completes tasks which stand out for their dimensionality, complex, non-uniform structure and a big amount of processed data. However, the final phase is the targeted one. In this phase the analysis of object properties is carried out within the nanometer range, so it can facilitate the development of new engineering methods for constructing various technical objects with the specified technical parameters.

Nanoscale electronics is a new and most promising direction for the development of hardware components of high-performance parallel architecture computing systems [2]. Obviously, the development of neural network computing devices and the next generation neural chips will be based on the homogeneous nanoscale hardware components and pulse frequency modulation with hybridization of the CMOS-technology and nanoscale electronic elements [3].

The theory and practice of engineering design of hardware implementations of neural network computing systems with the specified reliability parameters with the use of nanoscale electronic hardware components are being formulated and developed. One of the most crucial and unsolved problems is the development of methods for providing the required fault-tolerance level which is not achieved automatically within the specified tolerance, and in some cases it cannot be achieved in principle due to some physical features that show up within the nanometer range.

Thus, this research is mainly aiming to develop a general approach to analyzing the properties of nanoscale electronic elements used in the engineering design of neural computers as well as separate neural network and neuromorphic components in the data processing systems which possess maximum operation and fault tolerance reliability parameters. The methods based on this approach are designed to determine the correlation between the fault tolerance parameter of neural network devices based on nanoscale electronic elements and the internal and external factors which predetermine its alteration.

\section{METHODS}

The analysis of research and technology publications [4-8] enabled us to reveal some downsides in the existing, few in number methods for analyzing the properties of nanoscale electronic elements for their further application in the facilities with neural network architecture or the ones which operate in the neural network logical basis. They would help achieve maximum reliability parameters with regard to the operation and fault tolerance of the system in question.

Here we are proposing a new approach to solving this problem. This approach integrates the potential of the theoretical and experimental analysis of the properties of nanoscale electronic elements in order to provide main technical parameters of the neural network facilities designed on their basis.

The experimental part of the approach is expected to formulate and apply possible mechanisms for influencing artificially on various properties of the examined nanoscale objects which are capable of provoking the

\footnotetext{
*nauka-murom@yandex.ru
} 
system's reaction in the shape of alteration in the values of its parameters.

The theoretical part fulfils an analytical function and determines qualitative and quantitative dependencies between the change in the system element parameters and the properties of its elements which predetermine this change. The quantitative dependencies will help get the tolerance limits on the change of element parameters, and the qualitative will make it possible to determine mechanisms for influencing the physical properties of elements with the aim of optimizing the system's technical parameters.

Let us make use of the tools of system-oriented analysis in order to develop practical methods (based on the proposed approach) for analyzing the properties of nanoscale elements. According to our approach which integrates the theoretical and experimental research, the correlation of an introduced operational hypothesis with the experimental data of observations shall be considered as the basis of integrated analysis of the system. In this instance, the proposed approach constitutes a particular case of implementing a theory of experiment planning, where the experimentally obtained data about the system operation is the basis for theoretical calculations and deductive outcomes.

The classifications and terminology used in the proposed theoretical and experimental approach are regulated by a number of standards on the basis of which the methods for doing a theoretical and experimental research can be divided in accordance with the research goal and form of reporting the results. The methodology used in this study belongs to a quantitative experiment that both registers the result and determines the correlation between the quantitative characteristics of a phenomenon and the way of external action.

The factor, used for conducting such an experiment, is a result of the task that is being completed by the methods based on the given approach and it is the quantitative value of various properties of the nanoscale electronic elements which constitute the system. The variable parameter that serves as a reflection of various parameters of the optimized values is basically a multitude of factor levels.

The final object (fault tolerance parameter) depends statistically on a selected response factor. As the main numerical characteristic of the system's fault tolerance, a fixed accuracy level (with specified tolerance) or faults (errors) in the system's operation based on the results of experimental research will serve as the response.

The experiment has a searching (extreme) nature and is aimed at finding the correlation of levels of the controllable factors when an optimal (extremely maximum or minimum) value of the response function is achieved. The resultant dependency cannot be presented as a mathematical formula, however, it shows the extent of optimization potential and respectively the efficiency of the methods for engineering fault-tolerant neural network computing systems based on nanoscale electronic hardware components.

Availability of a multitude of factor levels is a necessary condition. This type of research belongs to the active experiment class. It has its own data set that determines the number, conditions and procedure of implementing tests. This fact proves that the proposed theoretical and experimental approach to analyzing the properties of nanoscale electronic elements as part of the parallel computing systems is based on the global and scientifically incontestable approach experimental design theory.

In the course of work we will develop some computational models of nanoscale electronic elements integrated into a system aimed at converting data and it has a characteristic which is of a particular interest for us accuracy of operation (errors of operation). The given models will be used for conducting an experiment and processing the research results.

The technology for analyzing the properties of a nanoscale object comes down to a number of procedures:

- synthesis of the computational models of nanoscale elements which meet the specified technology requirements;

- development of the system in the shape of a computational parallel architecture on the basis of the available limitations, in order to convert the data that has the best accuracy of operation;

- setting a goal of the experiment and making a plan of the experiment taking into account the tasks in hand; - quantitative estimation of the system's technical parameters and generation of statistical data showing alteration of element parameters where the levels of factor are different;

- analytical estimation and recognition of the desired dependencies of alterations in the system's accuracy of operation from alterations of the element parameters caused by the impact on their properties.

\section{RESULTS}

A crossbar array neural network computing architecture with the use of nanoscale elements - memristors acting as connection (synapses) among the neuron layers [8] was simulated as an experimental study of the methodology proposed in this research.

A synapse is an element that performs a weighed signal transfer (1) from the neuron of one layer to the neuron of the next layer within an artificial neural network (figure 1).

$$
f(x)=w \cdot x,
$$

where $f(x)$ - input of the neuron, $w$ - weights of the synapse, $x$ - output of the previous neuron.

\section{Inputs}

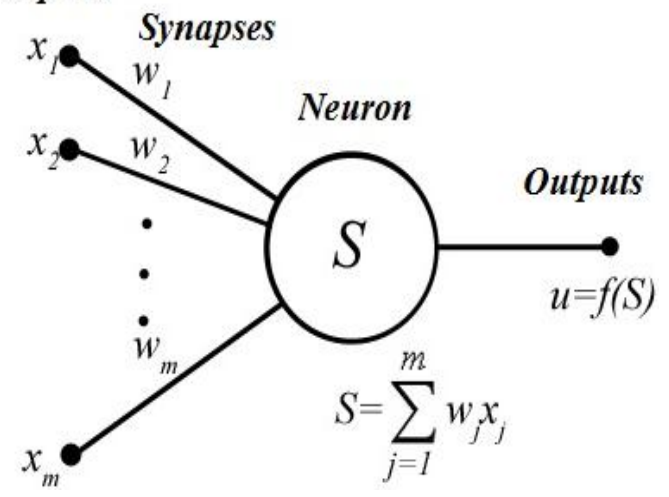

Fig. 1 - Artificial neuron scheme 


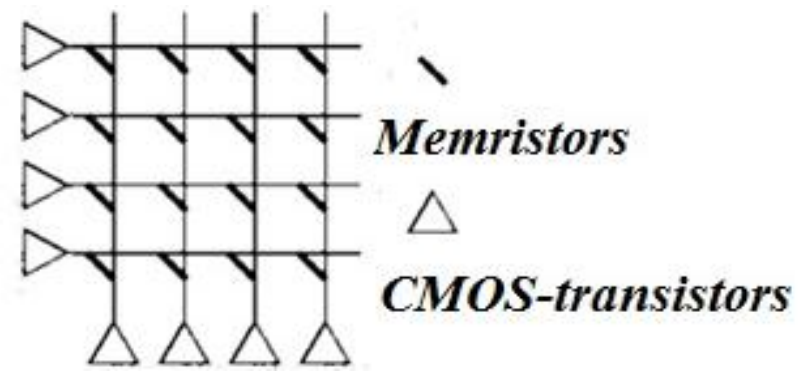

Fig. 2 - A fragment of the computing memristor crossbar array controlled by CMOS transistors

A memristor is a passive element in microelectronics; its resistance depends on a charge that has passed through it. When a voltage in the circuit is turned off, a memristor doesn't change its state and it registers the last resistance value [9]. The following equations describe the voltage-current characteristic (2) and resistance (3) of a memristor:

$$
\begin{gathered}
v(t)=\left(\frac{\operatorname{Ron} \cdot w(t)}{D}+\operatorname{Roff}\left(1-\frac{w(t)}{D}\right)\right) \cdot I(t), \\
R=\rho \cdot \frac{d}{S},
\end{gathered}
$$

where $v(t)$ - voltage, $I(t)$ - current, $w(t)$ - thickness of memristor's doped region, $D$ - total thickness of a memristor, Ron - the minimum resistance value of a memristor, Roff - the maximum resistance value of a memristor, $R$ - resistance, $\rho$ - resistivity of the material, $d$ - thickness of the active layer, $S$ - contact surface.

As for an environment for the experiment, we chose a tool for modeling and simulating physical objects the "Simscape» application software package that is controlled by the "Matlab». We synthesized simscape memristor models (figure 3) which constituted a twolayer feedforward neural network in the shape of a crossbar array; its schematic visualization is shown in figure 2. Its function is to approximate the differential equation (4):

$$
f^{\prime}(x)=a+b \cdot \ln (x) \text {. }
$$

The artificial neural network was trained with the help of Neural Network Toolboxes, after that the obtained weighing coefficients were recorded in the synapse model, so that the crossbar array could be used as a computing system. The system's maximum accuracy of operation was achieved when there were 17 neurons in the first layer and one output neuron in the second layer. The neuron activation functions - the hyperbolic tangent sigmoid transfer function in the first layer and the linear transfer function in the second one. The training algorithm - Levenberg-Marquardt backpropagation. Approximate error (sum squared error performance function) amounted to $8.52 \cdot 10^{-13}$.

We revealed a number of factors impacting the material properties: the top contact surface, the bottom contact surface and the thickness of the dielectric layer. The array was programmatically exposed to alteration of the qualitative value of the factors at various levels. On each stage of alteration of the properties of the memristors the qualitative alteration of the system's element parameters and the fault tolerance parameter of the system as a whole were registered.

The research findings show that the abovementioned physical phenomena and processes in nanoscale electronic elements which carry information in the simulated artificial neural network, determine the fault tolerance parameter of the system in question. This is due to the fact that the element properties impact their information parameters that determine the system's accuracy of operation. Therefore, analysis of the properties, which characterize the reliability of nanoscale electronic elements used for constructing data processing systems, based on the idea that data should be considered as an object for the conversion in the stated systems, is the most promising and it enables us to take into account all the factors that predetermine the reliability parameter under examination. Table 1 shows the general research results.

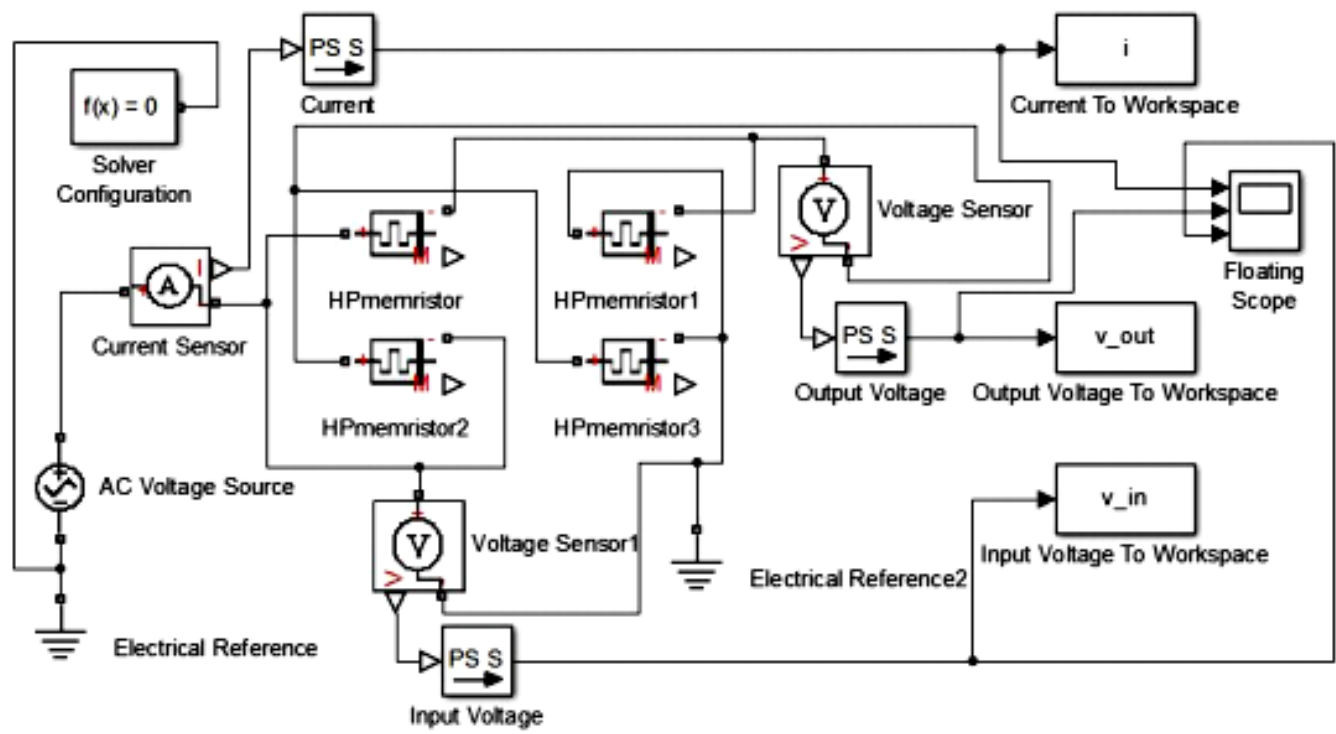

Fig. 3 - Neuron synapse based on a simscape memristor model 
Table 1 - The experimental results of studying the proposed methodology using the model of an artificial neural network approximating a deferential equation as an example

\begin{tabular}{|c|c|c|c|}
\hline $\begin{array}{c}\text { Factor affecting the } \\
\text { properties of memristor }\end{array}$ & $\begin{array}{c}\text { Changing the geo- } \\
\text { metrical parameter of } \\
\text { synapse }\end{array}$ & $\begin{array}{c}\text { Changing the infor- } \\
\text { mation parameter of } \\
\text { synapse }\end{array}$ & $\begin{array}{c}\text { Errors of functioning } \\
\text { the system } \\
\text { (SSE criterion) }\end{array}$ \\
\hline \multirow{2}{*}{$\begin{array}{c}\text { Upper contact surface of } \\
\text { memristor }\end{array}$} & $0 \mathrm{~nm}$ & $0 \%$ & $8.52 \cdot 10^{-15}$ \\
\cline { 2 - 4 } & $10 \mathrm{~nm}$ & $0.9 \%$ & $8.68 \cdot 10^{-15}$ \\
\cline { 2 - 4 } & $20 \mathrm{~nm}$ & $1.5 \%$ & $9.56 \cdot 10^{-15}$ \\
\hline \multirow{2}{*}{$\begin{array}{c}\text { Bottom contact surface of } \\
\text { memristor }\end{array}$} & $40 \mathrm{~nm}$ & $1.5 \%$ & $16.42 \cdot 10^{-15}$ \\
\cline { 2 - 4 } & $0 \mathrm{~nm}$ & $0 \%$ & $8.52 \cdot 10^{-15}$ \\
\cline { 2 - 4 } & $10 \mathrm{~nm}$ & $1.2 \%$ & $10^{-12}$ \\
\cline { 2 - 4 } & $20 \mathrm{~nm}$ & $1.7 \% \cdot 10^{-15}$ \\
\hline \multirow{2}{*}{$\begin{array}{c}\text { The thickness of memris- } \\
\text { tor's doped region }\end{array}$} & $40 \mathrm{~nm}$ & $2.1 \%$ & $12.55 \cdot 10^{-15}$ \\
\cline { 2 - 4 } & $0 \mathrm{~nm}$ & $0 \%$ & $8.52 \cdot 10^{-15}$ \\
\cline { 2 - 4 } & $2 \mathrm{~nm}$ & $8.6 \%$ & $5.62 \cdot 10^{-13}$ \\
\hline
\end{tabular}

\section{CONCLUSIONS}

In the course of the research we achieved its goal and met all the set targets. Here are some of the main findings:

- we proposed a theoretical and practical approach to the practical analysis of the properties of nanoscale electronic hardware components in order to determine the quantitative and qualitative correlation among them and technical parameters of the facilities made on their basis;

- we developed a methodology which makes it possible to determine the correlation between the fault

\section{REFERENCES}

1. Л.А. Зинченко, В.А. Шахнов, Инженерный журнал: наука и инновации. 6, 57 (2013) (L.A. Zinchenko, V.A. Shakhnov, Inzhenernyy zhurnal: nauka i innovatsii 6 , 57 (2013)).

2. А.И. Галушкин, Приложение к журналу «Информационные технологии». Condens. Matter. 3 (2012) (A.I. Galushkin, Prilozheniye $k$ zhurnalu "Informatsionnyye tekhnologii». Condens. Matter. 3 (2012)).

3. А.И. Галушкин, Инфорлаиионные технологи 4, 2 (2014) (A.I. Galushkin, Informatsionnyye tekhnologi 4, 2 (2014)).

4. S. Spiga, International Interconnect Technology Conference and Materials for Advanced Metallization Conference (IITC/MAM), 213 (Grenoble: IEEE: 2015). tolerance of a parallel computing system and a number of properties of nanoscale electronic elements that are present in the system in question.;

- we experimentally examined the proposed methodology and as a result we determined the characteristics of the nanoscale memristors, used as the synaptic links of a neural network computing system, which determine the fault tolerance parameter.

\section{ACKNOWLEDGEMENTS}

The reported study was funded by RFBR, according to the research project No. 16-37-60061 mol_a_dk.

5. R.P. Shukla, G. Jain, S. Chandra, A. Ranjan, Futuristic Trends on Computational Analysis and Knowledge Management (ABLAZE), 102 (New Delhi: IEEE: 2015).

6. D. Newberry, Nanotechnology (IEEE-NANO), 800 (Portland: IEEE: 2011).

7. C. Yakopcic, R. Hasan, T.M. Taha, National Aerospace and Electronics Conference (IEEE-NAECON 2014), 243 (Dayton: IEEE: 2014).

8. K.-H. Kim, S. Gaba, D. Wheeler, J.M. Cruz-Albrecht, T. Hussain, N. Srinivasa, W. Lu, Nano Lett. 12, 389 (2012).

9. L.O. Chua, IEEE T. Circuit Theory. 18 No 5, 507 (1971). 